\title{
Morphology Study of Phosphonated Peptoid Block Copolymer
}

\author{
Xi Jiang ${ }^{1}$, Jing Sun ${ }^{2,5}$, Ronald Zuckermann ${ }^{2}$, Kenneth H. Downing ${ }^{3}$ and Nitash Balsara ${ }^{1,4}$ \\ 1. Materials Science Division, ${ }^{2 .}$ Molecular Foundry, ${ }^{3 .}$ Molecular Biophysics and Integrated Bioimaging \\ Division, Lawrence Berkeley National Lab, Berkeley CA, 94720 \\ 4. Department of Chemical and Biomolecular Engineering, University of California, Berkeley, CA \\ 94720. \\ 5. Qingdao University of Science and Technology, Qingdao, China 266042
}

Proton-conducting soft materials have attracted considerable attention since they play an important role as electrolyte membranes in both fuel cells and artificial photosynthesis.[1] The most widely studied electrolyte membranes are based on sulfonated polymers such as Nafion. However, sulfonic acid-based membranes are ineffective proton transporters at high temperatures (above $80{ }^{\circ} \mathrm{C}$ ), since very little water is retained at high temperatures. Phosphonated polymers are attractive systems because they exhibit efficient proton transport under low water uptake conditions.[2] Herein we report the HAADF-STEM and Cryo-EM characterization of a sequence-defined phosphonated peptoid block copolymer (BCP): poly- $N$-(2-ethyl)hexylglycine-block-poly- $N$-phosphonomethylglycine (pNeh- $b$-pNpm). The structure of synthesized peptoid BCP is shown in Figure 1, and the synthesis details can be found elsewhere. [3]

In this work we studied the morphologies of BCP ultra thin films that had undergone various treatments in order to investigate the relationship between morphology and performance as electrolyte membrane. All thin films were produced by drop casting a $0.1 \mathrm{wt} \% \mathrm{MeOH} / \mathrm{THF}$ 50:50 solution on continuous carbon or lacey carbon films. Thin films were annealed in a humidity chamber using water vapor at $98 \%$ relative humidity and $25^{\circ} \mathrm{C}$ for 24 hours, followed by air drying. Hydrated samples were obtained by annealing the as-cast thin films in a Vitrobot at nominal settings of $100 \%$ humidity and $25^{\circ} \mathrm{C}$ and then plunging into liquid ethane after blotting. Self-assembled vesicles were prepared by dropping the solution into water. $\mathrm{MeOH}$ and THF were slowly evaporated subsequently at low vacuum. HAADFSTEM images in Figure 2 represent the morphologies of dry moisture-annealed thin films on continuous carbon supporting film (Figure 2a) and lacy carbon supporting film (Figure 2b) respectively. One can see honeycomb structures in Figure 2a with bright walls and bright center domains. The bright domains, which have higher atomic number, are the phosphorus-rich blocks in the peptoid. In contrast to the honeycomb structures on continuous carbon, Figure $2 \mathrm{~b}$ reveals lamellar microphase separation after water vapor annealing. The lamellar structure with similar domain spacing are confirmed in bulk by small angle X-ray scattering as well, suggesting that the freestanding thin film on lacey carbon has the same microphase separated structure as bulk film. In contrast, the morphology of thin films on continuous carbon is significantly affected by interfacial energy so that it presents the honeycomb structure. The vitreous hydrated thin film on lacey carbon was then imaged by cryo-EM after annealing in order to illustrate the percolating network of conductive channels. The cryo-EM image in Figure 2c suggests a honeycomb structure with phosphorus-rich domains as walls and cores. The phosphorus-rich domains are responsible for the proton transportation when the film is hydrated. These conductive channels are much larger and uniform than the percolating networks in Nafion.[3] It is surprising to see that the hydrated freestanding thin film has morphology similar to the water annealed thin film on carbon substrate. This interesting phenomenon will be studied in future work. Figure $2 \mathrm{~d}$ represents a self-assembled peptoid vesicle in water. This vesicle has a bilayer shell with water in the core, similar to liposomes and other polymersomes. Cryo-EM images suggest that the dark phase is the hydrophilic, 
phosphorus-rich block and the inner bright phase is the hydrophobic block.

By analyzing the HAADF-STEM images of phosphonated peptoid block copolymers, we conclude that the morphology is significantly influenced by interfacial energy during annealing. The lamellar morphology is a stable microphase separated structure in both thin films and bulk. The conductive channels have been illustrated in hydrated thin films. They show a much more uniform percolation network than Nafion. For the first time, pepoid vesicles have been prepared and imaged using cryo-EM. The structure of a bilayer membrane can be clearly identified. These morphological studies may lead to better understanding of the proton transportation mechanisms in phosphonated peptoid block copolymer.

\section{References:}

[1] Steele, B. C. H.; Heinzel, A. Nature 2001, 414, 345.

[2] Rikukawa, M.; Sanui, K. Prog. Polym. Sci. 2000, 25, 1463.

[3] Sun, J.; Zuckermann, N. R. ACS Nano, 2013, 7,4715

[4] Allen, F. I. et al., ACS Macro Lett. 2015, 4, 1

[5] Funding for this work was provided by the Soft Matter Electron Microscopy Program, supported by the Office of Science, Office of Basic Energy Science, U.S. Department of Energy, under contract no. DE-AC02-05CH1 1231. The work was carried out at the National Center for Electron Microscopy within the Molecular Foundry at Lawrence Berkeley National Laboratory, supported by the Office of Science, Office of Basic Energy Science, U.S. Department of Energy, under Contract No. DE-AC02$05 \mathrm{CH} 11231$.

Figure 1. Structure of block copolypeptoid $\mathrm{pNeh}_{\mathrm{n}}-\mathrm{b}-\mathrm{pNpm}_{\mathrm{m}} ; \mathrm{n}$ and $\mathrm{m}$ are the degrees of polymerization of pNeh and pNpm blocks, and they are 18 and 18 in this study respectively.
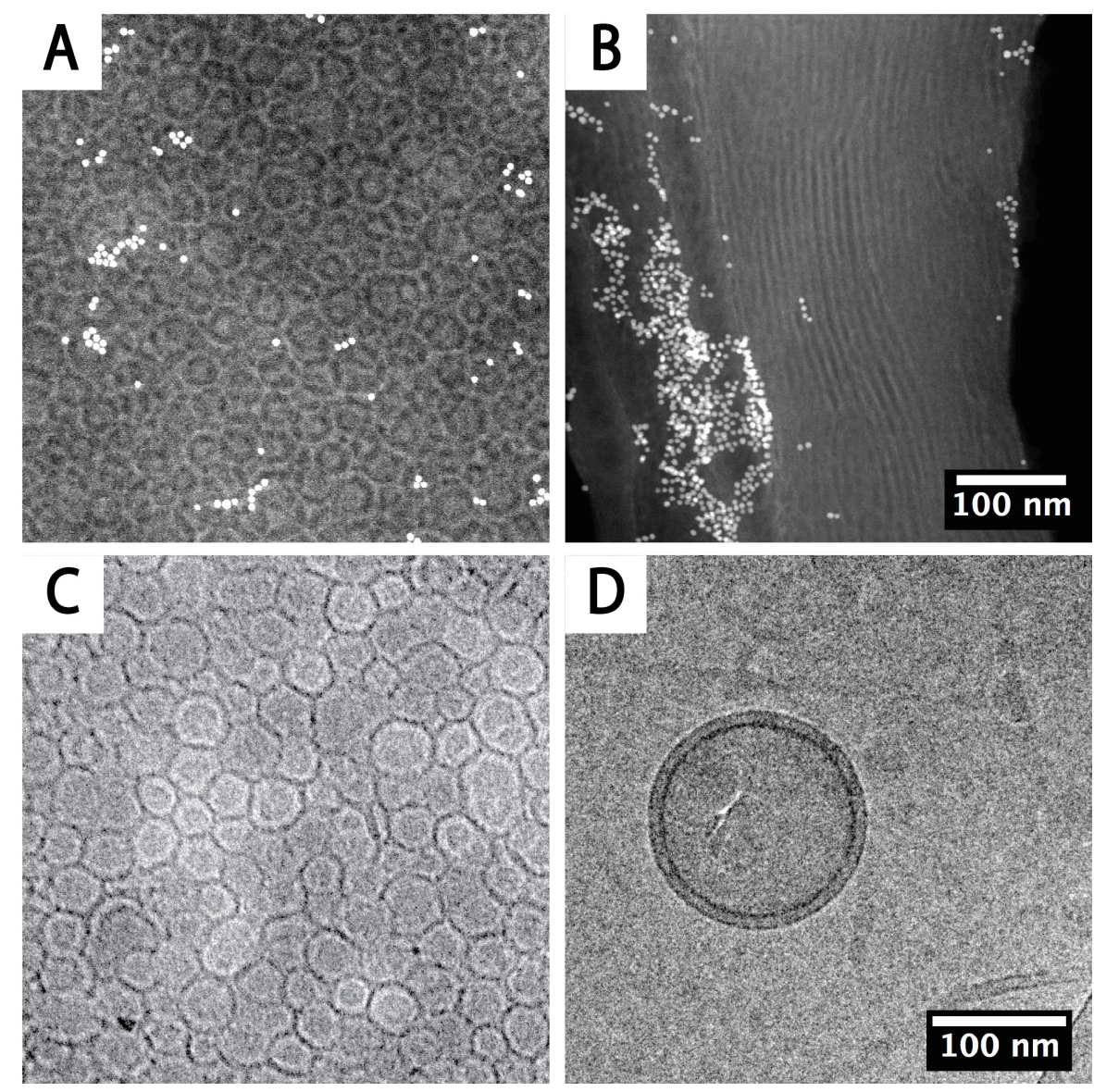

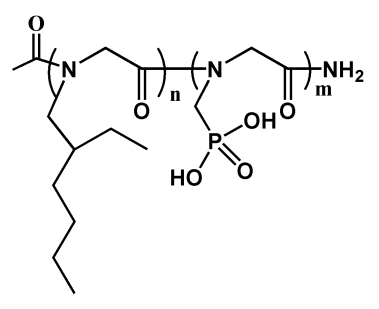

Figure 2. (a) HAADF-STEM image of thin film on continuous carbon annealed at high humidity and then air-dried; (b) HAADFSTEM image of freestanding thin film on lacey carbon annealed at high humidity and then air-dried; (c) Cryo-EM image of hydrated freestanding thin film on lacey carbon; (d) Cryo-EM image of self-assembled peptoid vesicle in aqueous solution. 